NBER WORKING PAPER SERIES

\title{
SOCIOECONOMIC STATUS AND MEDICAL CARE EXPENDITURES IN MEDICARE MANAGED CARE
}

\author{
Kanika Kapur \\ Jeanette A. Rogowski \\ Vicki A. Freedman \\ Steven L. Wickstrom \\ John L. Adams \\ José J. Escarce \\ Working Paper 10757 \\ http://www.nber.org/papers/w10757 \\ NATIONAL BUREAU OF ECONOMIC RESEARCH \\ 1050 Massachusetts Avenue \\ Cambridge, MA 02138 \\ September 2004
}

This research was supported by grants RO1-HS/AG09630 and PO1-HS10770 from the Agency for Healthcare Research and Quality The views expressed herein are those of the author(s) and not necessarily those of the National Bureau of Economic Research.

(C2004 by Kanika Kapur, Jeannette A. Rogowski, Vicki A. Freedman, Steven L. Wickstrom, John L. Adams, and José J. Escarce. All rights reserved. Short sections of text, not to exceed two paragraphs, may be quoted without explicit permission provided that full credit, including (C) notice, is given to the source. 
Socioeconomic Status and Medical Care Expenditures in Medicare Managed Care Kanika Kapur, Jeannette A. Rogowski, Vicki A. Freedman, Steven L. Wickstrom, John L. Adams, and José J. Escarce NBER Working Paper No. 10757

September 2004

JEL No. I1

\begin{abstract}
$\underline{\text { ABSTRACT }}$
This study examined the effects of education, income, and wealth on medical care expenditures in two Medicare managed care plans. The study also sought to elucidate the pathways through which socioeconomic status (SES) affects expenditures, including preferences for health and medical care and ability to navigate the managed care system. We modeled the effect of SES on medical care expenditures using Generalized Linear Models, estimating separate models for each component of medical expenditures: inpatient, outpatient, physician, and other expenditures. We found that education, income, and wealth all affected medical care expenditures, although the effects of these variables differed across expenditure categories. Moreover, the effects of these SES variables were much smaller than the effects found in earlier studies of fee-for-service Medicare. The pathway variables also were associated with expenditures. Accounting for the pathways through which SES affects expenditures narrowed the effect of SES on expenditures; however, the change in the estimates was very small. Thus, although our measures of preferences and ability to navigate the system were associated with expenditures, they did not account for an appreciable share of the impact of SES on expenditures.
\end{abstract}

Kanika Kapur

RAND

1700 Main Street

Santa Monica, CA 90407

kapur@rand.org

Jeannette A. Rogowski

RAND

Vicki A. Freedman

Policher Research Institute
Steven L. Wickstrom

Center for Health Care Policy and

Evaluation

United Health Care

John L. Adams

RAND

José J. Escarce

RAND and NBER

escarce@rand.org 


\section{INTRODUCTION}

Managed care enrollment among elderly Medicare beneficiaries increased dramatically during the 1980s and 1990s, from 3.5 percent of beneficiaries in 1985 to 16 percent in 2000 (CMS, 2002). The number of Medicare managed care enrollees has declined in recent years due to plan withdrawals from the Medicare+Choice market. Nonetheless, more than 5 million older Americans are currently enrolled in Medicare managed care plans.

Because managed care plans use financial incentives and administrative rules to limit utilization (e.g., Gold et al., 1995), policy makers have been concerned that Medicare managed care enrollees face barriers to receiving appropriate care. Several studies have shown that medical care utilization is lower among Medicare managed care enrollees compared to beneficiaries in the traditional Medicare fee-for-service (FFS) program (Manning et al., 1984; Miller and Luft, 1994, 1997; Chernew 1995), although rates of preventive services are higher in managed care (Tudor et al. 1998; Greene et al. 2001). Medicare beneficiaries with low socioeconomic status (SES) may be especially likely to face barriers to care under managed care (PPRC, 1996). However, there is little research on the role of SES in medical care utilization or expenditures among seniors in managed care.

Studies of the Medicare FFS program have found that seniors with low SES utilize medical care services less frequently than those with higher SES (e.g., Gornick, 1996; Mutchler and Burr, 1991; Hoenig et al., 1996; Escarce and Puffer, 1997; Miller et al., 1997). For example, Escarce and Puffer (1997) found sizable effects of household income and education on medical care expenditures and physician visits. Other studies found similar income and education effects on physician visits (Mutchler and Burr, 1991; Miller et al., 1997; Blustein and Weiss, 1998), and more pronounced effects for specialist physician visits (Blustein and Weiss, 1998). 
However, the applicability of this research to managed care may be limited by differences between the managed care and FFS environments that may modify the influence of SES on use. Under FFS Medicare providers have little incentive to constrain utilization, whereas in managed care settings providers are motivated to impose impediments to care. Because beneficiaries must learn to navigate the managed care system in order to overcome these impediments, education or the ability to learn may be more important in managed care than in FFS Medicare. On the other hand, financial barriers to care may be reduced in managed care, rendering financial resources less important.

The few studies that have examined the relationship between SES and medical care utilization in Medicare managed care have focused on preventive care. A national study of quality of care in Medicare managed care plans using HEDIS data found disparities in breast cancer screening by race, education, and income (Schneider et al., 2002). In another study based on the Medicare Current Beneficiary Survey, Schneider et al. (2001) found that white beneficiaries were more likely than blacks to receive influenza vaccinations, and that there was no difference in the magnitude of the disparity in Medicare managed care plans compared with FFS Medicare. In a study of Medicare managed care enrollees in four U.S. cities, Scott et al. (2002) found that black enrollees and enrollees with inadequate health literacy were less likely than their white and more literate counterparts ever to have received an influenza vaccination.

This paper contributes to the limited literature on the role of SES in Medicare managed care by examining the effects of SES on medical care expenditures in two Medicare managed care plans. Our study goes beyond previous research in three ways. First, we incorporate a comprehensive set of SES measures including household income, wealth, and educational attainment. Second, we fully characterize the role of SES in medical care expenditures by 
separately modeling various components of expenditures. Third, we attempt to assess the roles of the most likely pathways by which SES may affect utilization and expenditures.

\section{CONCEPTUAL FRAMEWORK}

We consider the effect of SES on the use of medical care by elderly persons in managed care plans in the context of the standard health production function model (Grossman, 1972). In this model, medical care is valued because it produces improvements in health, and the demand for medical care derives from the demand for health. The health production function translates the use of medical care services into improvements in health.

Within this framework, there are at least three pathways through which SES can affect medical care expenditures by Medicare managed care enrollees. First, SES may influence preferences for health and medical care versus the consumption of other goods and services, thereby affecting the demand for care. For instance, persons of low SES may have learned behaviors with respect to seeking care that are developed over a lifetime of limited access to the medical care system and manifest themselves as differences in preferences. Alternatively, persons with low SES may be less likely to be adequately informed about the benefits of good health or good medical care, and hence may have a lower demand for care (Scott et al., 2002). SES may also affect preferences for the type of medical care; for example, better educated persons may prefer receiving care from a specialist rather than a generalist.

Second, SES may affect the ability to navigate the managed care system to obtain the desired level of medical care. In the standard Grossman (1972) model, there are no supply-side constraints on the medical services patients receive. However, managed care settings are designed to control utilization through supply-side constraints, including restricting the network 
of providers, requiring the use of gatekeepers and utilization management (Gold et al., 1995). In such settings, patients may be unable to obtain the services they desire due to barriers erected by managed care plans (e.g., financial and nonfinancial incentives to providers, administrative hassles, etc.) and managed care enrollees who are more adept at navigating the system may be more likely to obtain the care they desire. The ability to navigate around these barriers may be related to SES (Scott et al., 2002); for instance, persons with higher educational attainment may be more adept in obtaining preauthorization for desired services or referrals to specialists.

Third, SES may affect utilization through the ability to pay for services. In managed care settings where cost sharing is low, however, ability to pay is likely to be less important than other factors.

Whereas the first pathway applies to both the managed care and FFS settings, the second and third pathways are most likely to generate differences between FFS and managed care with regard to SES effects on medical care expenditures. Ability to navigate the system, for example, may be more important in managed care than in FFS. On the other hand, managed care may help to blunt SES differences in medical care expenditures if the coordination of care through a primary care physician (PCP) that is typical of managed care reduces access barriers and informational asymmetries for individuals with low SES (Ettner, 1999; Williams et al., 2001; Sarver et al., 2002). Managed care may also allocate services more efficiently through utilization management and utilization guidelines, thus narrowing the SES gap in expenditures. On net, the effects of SES on medical care expenditures in managed care are ambiguous, and depend on the main pathways underlying these effects and on the extent to which managed care features exacerbate or blunt them. Therefore, the effects of SES on expenditures must be empirically determined. 


\section{METHODS}

\section{Setting}

Both health plans in this study have Medicare risk contracts and participate in the Medicare+Choice program. Both plans are independent practice association (IPA)-model HMOs that contract with health care providers in their communities to create provider networks. One plan is located in a Northeastern metropolitan area, the other in the Midwest. Enrollees in both plans are required to select a PCP; however, enrollees in the Northeastern plan are required to obtain PCP referrals for specialty care, whereas enrollees in the Midwestern plan are not required to do so. At the time of the study, the copayment for an office visit was $\$ 15$ in the Northeast plan and \$10 in the Midwest plan. Other benefits were similar in both plans, and both provided limited coverage for prescription drugs. ${ }^{1}$ Both plans pay PCP and specialist physicians discounted fee-for-service, and PCPs face no financial incentives to limit referrals.

\section{Subjects}

The target population for our study consisted of 20,227 aged Medicare beneficiaries (i.e., 65 years or older) enrolled in the two study plans. We used stratified random sampling to increase the number of low-income enrollees in the study sample. For each study plan, one

\footnotetext{
${ }^{1}$ Both plans had zero premiums, a 20 percent outpatient hospital coinsurance, and a $\$ 50$ co-payment for emergency room visits, which were waived if the patient was admitted to the hospital. In the Midwest plan, the inpatient copayment was $\$ 350$ per admission to a maximum of $\$ 700$ annually. In the Northeast plan, the inpatient copayment was $\$ 75$ per day to a maximum of $\$ 750$ annually.
} 
stratum consisted of enrollees who were dually eligible for Medicaid in addition to Medicare.

The remaining enrollees in each plan were assigned to a low-income stratum if they resided in a zip code where the majority of households had incomes less than twice the federal poverty line, according to the 1990 census, and to a high-income stratum otherwise.

We oversampled dually eligible enrollees and enrollees in the low-income strata, choosing sampling probabilities to obtain roughly equal numbers of enrollees from each plan. The resulting sample consisted of 6,996 enrollees, including 942 dually eligible enrollees, 700 enrollees in low-income zip codes, and 5,354 enrollees in high-income zip codes

\section{Data Sources}

The data sources for the study were administrative files from the study plans and a telephone survey of plan enrollees. The administrative data consisted of enrollment files, provider claims and facility claims for all services provided to enrollees in the initial study sample between April 1, 2000 and October 31, 2001. Enrollment files from the study plans were used to obtain each person's dates of enrollment, age, sex, and dual eligibility for Medicaid. Facility claims files were used to identify services provided by institutional providers, including hospitals, ambulatory surgery centers, skilled nursing facilities, hospices, laboratories, diagnostic imaging centers, home health care providers, and medical equipment providers; the type of facility that provided each service; and the payment for each service. Provider claims files were used to identify services provided by physicians and by nonphysician providers, the specialty of the physician or type of nonphysician provider who provided each service, and the payment for each service. Services were identified using Centers for Medicare and Medicaid Services Common Procedure Coding System (HCPCS) codes (similar to Current Procedural Terminology 
[CPT] codes) (AMA, 2000). We did not include prescription drug expenditures in this study because of incomplete claims data.

The survey included modules on demographic and socioeconomic characteristics, health status, family and household structure, attitudes and beliefs regarding medical care, current experience with medical care, and lifetime experience with health insurance and care. The module on demographic and socioeconomic characteristics elicited information on sex, race and ethnicity, educational attainment, household income, and household wealth (Smith, 1997). ${ }^{2}$ Health status was assessed using the Medical Outcomes Study 12-Item Short-Form Health Survey (SF-12), which yields summary measures of physical health (Physical Component Summary, or PCS) and mental health (Mental Component Summary, or MCS) that range from 0 to 100 , with means of 50 and standard deviations of 10 in the general population (Ware et al., 1996). Other measures of health status included self-rated general health (excellent, very good, good, fair, poor), self-rated memory and ability to make decisions, smoking history, and medical conditions and symptoms. The module on family and household structure elicited information on marital status, number and sex of children and number living in the household, and whether the enrollee lived with a spouse or partner.

The module on attitudes and beliefs used a 3-item scale to assess respondents' perceived "health locus of control," a construct from social learning theory that refers to whether health and health outcomes are under respondents' personal control or the control of medical care providers (Lau and Ware, 1981; Marshall et al., 1990). Each item was scored from 1 to 3, with higher scores reflecting a stronger belief in the efficacy of self-care versus formal medical care. We constructed a summary score by adding the scores from the 3 items in this module.

\footnotetext{
${ }^{2}$ Wealth was calculated as the sum of the current worth of the following assets: real estate; checking and savings accounts; certificates of deposits, government savings bonds, and treasury bills; Individual Retirement Accounts and Keogh plans; and stocks and mutual funds.
} 
The module on current experience with medical care included questions on how easily respondents were able to make appointments, contact providers, and obtain referrals.

The module on lifetime experience with health insurance and medical care elicited information on respondents' cumulative years of insurance coverage before Medicare (i.e., between 18 and 64 years of age), whether they had a usual source of medical care before Medicare (and the type of usual source), and whether they ever belonged to a health maintenance organization (HMO) before their current enrollment in a study plan.

The survey was conducted between April and October of 2000. A total of 528 enrollees in the initial study sample were ineligible because they were no longer enrolled in a study plan when they were contacted. Of the remaining 6,468 enrollees, 4,600 completed the survey for a 71 percent response rate.

\section{Variables}

Medical care expenditures were defined as the sum of all paid amounts reported in the provider and facility claims in the year following the survey administration for each respondent. Expenditures included plan payments and patient out-of-pocket payments. However, patient outof-pocket expenditures on non-covered services were excluded from medical care expenditures because we did not have claims data for these services.

We decomposed total medical care expenditures into 4 categories by type or location of service: (1) expenditures for physician services at any location, including inpatient, office-based settings, clinics, or other outpatient settings; (2) expenditures for inpatient hospital stays, excluding physician fees; (3) expenditures for outpatient hospital services, excluding physician fees; and (4) expenditures for all other services, including services rendered by nonphysician 
providers (e.g., chiropractors, optometrists, podiatrists, and physical therapists), home health care services, medical equipment and other medical services and supplies, ambulance services, and others. We further disaggregated expenditures for physician services into expenditures for primary care physician services and for specialist services. ${ }^{3}$

The key SES variables in our analysis consisted of three categories of educational attainment (less than high school, high school graduate, any college); four categories of household income based on percent of the federal poverty level $(<100$ percent of poverty, 100200 percent, 200-400 percent, $>400$ percent); and three categories of household wealth based on tertiles of the distribution of wealth across the men and women in the sample $\left(0-33^{\text {th }}\right.$ percentile, $33-67^{\text {th }}$ percentile, $67-100^{\text {th }}$ percentile). (We explored finer divisions of the SES variables, but found that the new adjacent categories this created had similar effects on expenditures.)

Additional enrollee characteristics used in the study included indicator variables for five age categories $(65-69,70-74,75-79,80-84, \geq 85)$; an indicator variable for female sex; three categories of race or ethnicity (non-Hispanic white, non-Hispanic black, “other”); and four marital status categories (married, widowed, divorced or separated, never married). Health status was measured by the PCS and MCS scores, self- rated general health, whether the enrollee had ever smoked, whether the enrollee died during the study period, and a full set of medical condition indicators. ${ }^{4}$ All models also included indicator variables for whether a proxy respondent was used during the interview, for whether the enrollee was dually eligible for Medicaid, and for the Northeast plan.

\footnotetext{
${ }^{3}$ Specialist categories included allergist, dermatologist, neurologist, neurosurgeon, opthalmologist, orthopedist, otolaryngologist, plastic surgeon, colon and rectal surgeon, psychiatrist, general surgeon, thoracic surgeon, urologist, cardiologist, gastroenterologist, hematologist, nephrologist, rheumatologist, endocrinologist, oncologist, vascular surgeon, pulmonologist, infectious disease specialist, neonatologist, and rehabilitation medicine.

${ }^{4}$ Medical condition indicators included in the analysis were high blood pressure, diabetes, cancer, lung disease, heart attack, congestive heart failure, angina, stroke, arthritis, ulcers, bowel disease, glaucoma, and depression. In addition, we included an indicator variable for unexplained weight loss.
} 
We also constructed several variables to assess the most likely pathways through which SES may affect medical care expenditures under managed care: preferences and ability to navigate the managed care system. We measured preferences for medical care by enrollees' attitudes and beliefs regarding care, using the 3-item summary score described earlier. Other proxy variables for preferences captured aspects of enrollees' lifetime experiences with the medical care system that could have shaped their care-seeking behavior, including health insurance coverage before Medicare, having had a usual source of care before Medicare, and the type of usual source of care before Medicare (e.g., doctor's office, clinic, hospital, etc.)

We measured enrollees' ability to navigate the managed care system by their reported ease of making an appointment, ease of contacting a provider, and whether they were able to obtain needed referrals to specialists. Additional variables to assess this pathway included whether enrollees had belonged to an HMO before enrolling in the study plan, which could have enabled them to become familiar with how managed care works; enrollees' memory and ability to make decisions; number of children; and whether enrollees lived alone. ${ }^{5}$ Children as well as a spouse or partner could help enrollees make appointments, make requests from providers, and understand and interpret plan rules, among other things.

We recognize that these measures are not a comprehensive set of all pathway variables, and that each measure may conceivably operate through more than one pathway. For example, belonging to an HMO may not only help enrollees learn how to navigate the system, but may shape their preferences for care as well. Nonetheless, we assumed that these measures would provide some insight into the mechanisms underlying SES effects on expenditures.

\footnotetext{
${ }^{5}$ Living with a family member could also affect utilization because the family member may serve as a source of informal care.
} 


\section{Regression Analyses}

To assess the effect of SES on medical care expenditures, we conducted multivariate regression analyses with expenditures in each of the 4 categories discussed earlier as dependent variables. For each category of expenditures, we estimated two sets of regression models. The first set of models estimated the effect of SES on medical care expenditures, adjusting for age, sex, marital status, race, health status, dual Medicaid eligibility, proxy status, and study plan as covariates. These models are similar to those used in earlier studies of SES and medical care utilization or expenditures (e.g., Escarce and Puffer, 1997; Miller et al., 1997). However, we also estimated a second set of models in which we added the pathway variables as covariates to assess whether the pathways accounted for the effects of SES.

Multivariate analyses for expenditures for inpatient and outpatient hospital stays were based on the two-part model of medical care utilization (Manning et al., 1981, 1987; Blough et al., 1999). The first part of the two-part model is an equation for whether a person has nonzero expenditures in a particular category during the year, and was specified as a probit model. The second part of the two-part model is an equation for the level of expenditures in the particular category conditional on nonzero expenditures, and was specified as a generalized linear model with a logarithmic link function and variance proportional to the square of the mean (McCullagh and Nelder, 1989; Blough et al., 1999; Manning and Mullahy, 2001).

Multivariate analyses for physician services expenditures and expenditures for other services were based on a one-part model for the level of expenditures. Ninety-six percent of enrollees had physician expenditures and 90 percent had other expenditures; therefore, the onepart model was the more suitable model for these components. The one-part model was specified 
as a generalized linear model with a logarithmic link function and with variance proportional to the square of the mean. (Using two-part models for physician services expenditures and other expenditures gave similar results, but the estimates were less precise.)

All analyses were estimated using weights that reflected both the sample design and survey nonresponse.

\section{Simulations}

We used simulations to obtain the predicted annual expenditures per person, in each category of expenditures, for each SES category, adjusted for other factors that may affect medical care expenditures and that were included as explanatory variables in the models. Each simulation for the estimates based on two-part models was conducted in three steps.

First, we used the estimated coefficients from the first part of the two-part model to predict the probability of nonzero expenditures for each person $i, E\left[p_{i}\left(\operatorname{Exp}_{i}>0\right)\right]$, for each $S E S$ category, by substituting the person's covariate values and alternately switching each indicator variable for SES category on and off. Similarly, we used the estimated coefficients from the second part of the two-part model to predict conditional expenditures (i.e., conditional on nonzero spending) for person $\mathrm{i}, \mathrm{E}\left(\operatorname{Exp}_{\mathrm{i}} \mid \operatorname{Exp}_{\mathrm{i}}>0\right)$, for each SES category.

Second, we predicted unconditional expenditures for person i, $\mathrm{E}\left(\operatorname{Exp}_{\mathrm{i}}\right)$ for each $\mathrm{SES}$ category, as:

$$
\mathrm{E}\left(\operatorname{Exp}_{\mathrm{i}}\right)=\mathrm{E}\left[\mathrm{p}_{\mathrm{i}}\left(\operatorname{Exp}_{\mathrm{i}}>0\right)\right] \mathrm{x} \mathrm{E}\left(\operatorname{Exp}_{\mathrm{i}} \mid \operatorname{Exp}_{\mathrm{i}}>0\right)
$$

Third, for each SES category, we averaged the individual predictions of unconditional expenditures across all the persons in the study sample. We used the delta method (Bishop et al., 
1975) to derive the standard errors of the predicted annual expenditures per person and the statistical tests of differences in expenditures across the SES categories.

Analogously, each simulation for the estimates based on one-part models was conducted in two steps. First, we used the estimated coefficients from the model to predict expenditures for person $\mathrm{i}, \mathrm{E}\left(\mathrm{Exp}_{\mathrm{i}}\right)$, for each SES category, by substituting the person's covariate values and

alternately switching each indicator variable for SES category on and off. Second, we averaged the individual predictions of unconditional expenditures across all the persons in the study sample and used the delta method to derive standard errors for these estimates.

We obtained predicted annual total medical care expenditures per person, for each of the SES categories, by summing the predicted expenditures across the 4 categories of expenditures. Standard errors of predicted total expenditures per person and statistical tests of differences in total expenditures across SES categories were obtained using a bootstrapping technique (Efron, 1982). The paper presents the findings of the simulations. Full regression results are available from the authors on request.

\section{RESULTS}

\section{Descriptive Data}

Total medical care expenditures averaged \$5200, with inpatient hospital expenditures accounting for 41 percent (\$2131), physician expenditures for 28 percent $(\$ 1471)$, outpatient hospital expenditures for 12 percent $(\$ 622)$, and expenditures on the other services for 19 percent (\$976). 
Table 1 presents descriptive data on SES and other sample characteristics. All statistics are weighted using sampling weights. Thirty-eight percent of the sample had less than a high school education, while 25 percent had some college education. By definition, one-third of the sample fell in each household wealth tertile. Eleven percent of the sample had household incomes below the poverty line, 44 percent of the sample was low-income (100-200 percent of poverty), and 13 percent was high-income (400 percent of poverty and higher).

Table 2 presents means of the pathway variables. Many of these variables were significantly associated with the measures of SES (data not shown). Thus higher income enrollees had stronger beliefs in the efficacy of formal medical care than their lower income counterparts. Higher educational attainment, household wealth, and household income were positively associated with having health insurance coverage, having HMO experience, and having a usual source of care, especially a doctor's office or clinic, before Medicare. For instance, only 72 percent of poor enrollees had a usual source of care between the ages of 18 and 64, compared with 92 percent of high-income enrollees. Sixty four percent of individuals in the top tertile of assets were insured all the time during age 18 to 64 compared with 47 percent in the bottom tertile. Enrollees with higher education also found making an appointment with a provider easier: 26 percent of enrollees with a high school degree reported that they found making an appointment very easy compared with 29 percent of enrollees with a college degree.

Higher educational attainment, household wealth, and household income were associated with better self-rated memory and ability to make decisions. For example, 15 percent of individuals with less than a high school degree rated their memory as excellent compared to 21 percent of individuals with a college degree. Conversely, low-income and less educated enrollees were more likely than their high-income peers to be living alone and to be dually 
eligible for Medicaid. Forty three percent of individuals with less than a high school degree were living alone compared with 37 percent of individuals with a college degree. The number of children was unassociated with SES.

\section{Regression Analyses}

\section{Effects of SES on Expenditures}

Table 3 reports predicted annual medical care expenditures, by expenditure category and SES, adjusted for differences in the covariates. The analyses revealed that higher educational attainment increased physician services expenditures. Enrollees with less than high school education had lower physician expenditures than enrollees with a high school degree or some college. However, the differences in physician expenditures by education did not result in statistically significant differences in total medical expenditures by educational attainment.

Household income had a large effect on medical care expenditures. Specifically, poor enrollees had substantially higher total medical expenditures than low-income or middle-income enrollees, and this effect was entirely due to inpatient hospital expenditures. The opposite pattern was found for expenditures for other services, where the poor had lower expenditures than all other income categories. Income had no effect on physician services expenditures.

The effect of household wealth on medical expenditures was mixed. Enrollees with high assets had higher total medical care expenditure than enrollees with medium assets. In addition, enrollees with high assets had higher physician services expenditures than enrollees with low or medium assets. For outpatient hospital expenditures and expenditures for other services, however, enrollees with low assets had higher expenditures than enrollees with medium assets.

Table 4 reports predicted expenditures by SES for two components of physician expenditures: expenditures for PCP services and expenditures for specialist services. 
Disaggregating physician services expenditures into these components revealed that the effects of SES observed in Table 2 were driven by specialist expenditures. In particular, enrollees with less than high school education had lower expenditures for specialist services than high school graduates and enrollees with some college, whereas we found no education differences for PCP services. Similarly, enrollees with high assets had higher specialist expenditures than enrollees with low or medium assets, but we found the opposite pattern for PCP expenditures. Enrollees with low assets had higher PCP expenditures than enrollees with medium assets, although the magnitude of the difference was small.

The effects of the covariates included in the first set of analyses are reported in the Appendix. In general, these variables had the expected effects on expenditures.

\section{The Pathway Variables and Expenditures}

In our second set of analyses, we reestimated the expenditure models after including the pathway variables as additional covariates. We found that our measures of preferences for medical care were significantly associated with medical care expenditures (Table 5). Thus a weaker belief in the efficacy of formal medical care, as quantified by a decrease in the care attitudes summary score from the $25^{\text {th }}$ to the $75^{\text {th }}$ percentile of the distribution in the sample, was associated with a $\$ 685$ decrease in total medical care expenditures. Enrollees who were insured only some or none of the time between the ages of 18 and 64 spent $\$ 1,034$ more on medical care than enrollees who were insured most or all of the time. Enrollees whose usual source of care between the ages of 18 and 64 was a hospital or emergency room incurred expenditures for other services that were $\$ 231$ higher than enrollees with no usual source of care.

Several of our measures of ability to navigate the managed care system were also significantly associated with medical care expenditures (Table 5). Enrollees who reported that 
getting appointments with providers was easy had total expenditures that were $\$ 698$ higher than enrollees who reported difficulty getting appointments. Enrollees with 1 to 10 years of HMO experience had total expenditures that were \$902 higher than enrollees with no HMO experience, although, curiously, enrollees with more than 10 years of HMO experience had expenditures similar to those with no experience. Enrollees who reported poor decision-making skills had total medical expenditures that were $\$ 362$ lower than enrollees who reported good decisionmaking skills.

The effect of the pathway variables was not restricted to particular components of medical expenditures. Even inpatient use, which arguably should be the least responsive to preferences and accessibility, was significantly associated with care attitudes, ease of making an appointment, HMO experience, and decision-making skills. Other pathway variables such as number of children, ease of contacting providers and self-rated memory obtaining referrals were not significant in the models, and were dropped from the final specifications. ${ }^{6}$

\section{The Pathway Variables and SES Effects on Expenditures}

Despite the fact that many of the pathway variables were associated with expenditures, including the pathway variables in the regression models did not appreciably narrow the SES differences in Table 3. To illustrate, Table 6 presents ratios of predicted expenditures for different SES categories derived from models with and without the pathway variables. For instance, without adjusting for pathway variables, the ratio of total medical expenditures for individuals with a high school degree relative to individuals without a high school degree was

\footnotetext{
${ }^{6}$ Although unrelated to the pathways we assessed, we also examined whether owning a car was associated with medical care expenditures, since car ownership was associated with SES. However, car ownership was not significant in the models.
} 
1.09 (Table 6). This ratio narrowed to 1.06 when we included the pathway variables. The results in the table indicate that the pathway variables explained a small share of the educational differences in medical care expenditures; however, these variables explained none of the expenditure differences based on household income or wealth.

\section{DISCUSSION}

This study examined the role of SES in medical care expenditures in two Medicare+Choice managed care plans. Our analyses found several significant effects of SES on expenditures. Additionally, we found that the impact of SES on expenditures varied according to the category of expenditures, substantiating the need for modeling the components of medical care expenditures separately.

We found that higher educational attainment and higher assets increased physician services expenditures, particularly expenditures on specialist services, and that higher assets increased total medical care expenditures as well. However, the effect of assets on outpatient expenditures and expenditures for other services was mixed. We also found that poor enrollees had substantially higher inpatient hospital expenditures and, as a result, higher total expenditures than nonpoor enrollees.

A possible mechanism for our finding that higher educational attainment increased specialist expenditures is that education may lead to a greater understanding of the benefits of specialist care. Alternatively, more highly educated enrollees may be better able to navigate the managed care system in order to obtain specialist referrals. It is also possible that physicians treat highly educated patients differently, regardless of managed care, and are more likely to satisfy 
these patients' requests for referrals or to believe that these patients are more capable of adhering to complex treatment regimens (e.g., Goldman and Smith, 2002).

Our finding that poor enrollees had substantially higher inpatient hospital expenditures than the nonpoor, even after adjusting for health status, is consistent with earlier research on both Medicare and non-Medicare populations showing that people with low SES have higher hospital use (Epstein et al. 1988; Epstein et al., 1990). The poor may prefer inpatient care, or their physicians may be more likely to admit them for inpatient care, if they have less support than the nonpoor for treatment and recovery at home. If the poor are more likely than the nonpoor to delay ambulatory care, they may develop more severe health conditions that require inpatient admission more frequently. Notably, Stern et al. (1991) found that low-income patients were more likely than their higher income counterparts to be admitted to hospitals through the emergency department. The poor may also have worse health status than the nonpoor in ways that were not captured by our health status measures. However, unobserved differences in health status would be expected to affect expenditures in all categories.

Our finding that higher assets increased physician services expenditures and total expenditures is consistent with previous studies that found positive effects of financial resources on physician visits and medical care expenditures (Escarce and Puffer, 1997; Mutchler and Burr, 1991; Miller, 1997; Blustein and Weiss, 1998). Earlier studies have used income as the sole measure of financial resources. Since wealth is a better indicator than income of financial resources among the elderly (Smith, 1997), however, it is not surprising to find a positive effect of wealth on expenditures without an effect of income. On the other hand, given the low level of cost sharing in the two study plans, wealth effects on expenditures may reflect differences in preferences for medical care rather than financial resources. If wealthier people have developed 
preferences for higher quality or "luxury" goods, these preferences may translate into medical care as well. Our finding that higher assets increased specialist expenditures is consistent with this interpretation. It is also possible that physicians treat wealthier patients differently and are more likely to refer these patients to specialists.

Notably, the effects of SES on medical care expenditures in this study were modest in size. In fact, these effects were smaller, or even opposite in direction, when compared with the SES effects observed in previous studies of FFS Medicare. For example, using 1987 data for a nationally representative sample of Medicare beneficiaries, Escarce and Puffer (1997) found that total medical care expenditures for high-income seniors were 24 percent higher than expenditures for poor seniors. In the current study, poor enrollees had the highest total expenditures, and expenditures did not differ across the other income categories. Similarly, Escarce and Puffer (1997) found that total expenditures for beneficiaries with some college were 23 percent higher and for high school graduates 13 percent higher than expenditures for beneficiaries who did not have a high school degree. The current study found corresponding differences in total expenditures of 13 percent and 9 percent, respectively.

It is possible that features of the Medicare managed care plans in this study contributed to blunting SES differences in expenditures. The study plans did not have financial incentives for providers to limit referrals and required all enrollees to select a PCP. Having a PCP may foster regular patient-provider relationships, which may reduce access barriers and promote use of needed services, especially by low-income people (e.g., Scott et al., 2002). Consistent with this notion, SES was unrelated to enrollees' reported ease of getting appointments, contacting providers, or obtaining referrals. 
Unfortunately, our exploration of the pathways through which SES affects medical care expenditures met with limited success. Although attitudes and beliefs regarding medical care, history of health insurance coverage, usual source of care before Medicare, ease of making an appointment, HMO experience, and decision-making skills were significantly associated with expenditures, including these variables in the regression models did not appreciably narrow the SES effects. Thus, while our findings suggest that preferences and ability to navigate the system influence spending, we were unable to uncover evidence that these are the main pathways underlying the effects of SES.

Our study has several limitations. First, we examined the experience of seniors from only two HMOs; therefore, our findings may not be generalizable across health plans and areas of the country. Second, although our measures of health status were comprehensive, unobserved differences in health status may have influenced our results. For example, we had no information on the severity of medical conditions. Third, as a result of incomplete pharmacy claims, we could not assess the impact of SES on prescription drug expenditures.

Finally, our ability to determine the role of alternative pathways may have been undermined by the fact that our pathway measures were limited. Thus, for instance, while we had a measure of beliefs in the efficacy of formal medical care, we had no direct measures of preferences or habits regarding the consumption of higher quality or "luxury" goods. Similarly, we used information on household and family structure, rather than direct measures, to assess support at home, and we had no measures of health knowledge, ability to comply with medical regimens, or treatment decisions by medical care providers. 
Despite these limitations, this study makes an important contribution to our understanding of the impact of SES on medical care expenditures in Medicare managed care. In particular, our results show a smaller effect of SES on medical expenditures in Medicare managed care compared with the effects in the existing literature based on traditional FFS Medicare. Coordination of care by PCPs, utilization management, use of practice guidelines and other quality assurance mechanisms, and other features of managed care plans may have helped attenuate SES disparities. If our findings are generalizable to other Medicare managed care plans, this would provide strong evidence for managed care as an instrument to foster equity in health care delivery (Chen and Escarce, 2004).

However, our study also suggests that research is needed to explore further the pathways or mechanisms by which SES affects medical care utilization, because developing effective policy interventions requires such an understanding. Better and more comprehensive measures of preferences, ability to navigate the system, health knowledge, and other potential pathways could be very fruitful in this regard. 


\section{REFERENCES}

American Medical Association. 2000. Physicians' Current Procedural Terminology. Chicago, IL: American Medical Association.

Blough, D.K., C.W. Madden, and M.C. Hornbrook. 1999. Modeling risk using generalized linear models. Journal of Health Economics 18:153-171.

Blustein, J., and L.J. Weiss. 1998. Visits to specialists under Medicare: socioeconomic advantage and access to care. Journal of Health Care for the Poor and Underserved. 9(2):15369.

Centers for Medicare \& Medicaid Services. 2002. Program information on Medicare, Medicaid, SCHIP and other programs of the Centers for Medicare \& Medicaid Services. Available online: http://www.cms.hhs.gov/charts/series/sec2.pdf. Accessed November 26, 2002.

Chen, A.Y., and J.J. Escarce. 2004. Quantifying income-related inequality in health care delivery in the United States. Medical Care 42(1): 38-47.

Chernew, M. 1995. HMO use of diagnostic tests: a review of the evidence. Medical Care Research and Review 52:196-222.

Efron, B. 1982. The Jackknife, the Bootstrap, and Other Resampling Plans. Philadelphia, PA: Society for Industrial and Applied Mathematics.

Epstein, A.M., R.S. Stern, and J. Tognetti et al. 1988. Association of patients' SES with the length of hospital stay and hospital charges within diagnosis-related groups. New England Journal of Medicine 318(24):1579-1585.

Epstein, A.M., R.S. Stern, and J.S. Weissman. 1990. Do the poor cost more? A multihospital study of patients' socioeconomic status and use of hospital resources. New England Journal of Medicine. 322(16):1122-8.

Escarce, J.J., and F.W. Puffer. 1997. Black-white differences in the use of medical care by the elderly: a contemporary analysis. In: Martin LG, Soldo BJ (eds). Racial and Ethnic Differences in the Health of Older Americans. Washington, D.C.: National Academy Press; 183-209.

Ettner, S.L. 1999. The relationship between continuity of care and the health behaviors of patients: does having a usual physician make a difference? Medical Care 37(6):547-55.

Gold, M.R., R. Hurley, and T. Lake et al. 1995. A national survey of the arrangements managed care plans make with physicians. New England Journal of Medicine 333:1678-1683.

Goldman, D.P., and J.P. Smith. 2002. Can patient self-management help explain the SES health gradient? Proceedings of the National Academy of Sciences 99(16): 10929-34. 
Gornick, M.E., P.W. Eggers, and T.W. Reilly et al. 1996. Effects of race and income on mortality and use of services among Medicare beneficiaries. New England Journal of Medicine 335:791-799.

Grossman, M. 1972. On the concept of health capital and the demand for health. Journal of Political Economy 80:223-255.

Greene, J., J. Blustein, and K.A.Laflamme. 2001. Use of Preventive Care Services, Beneficiary Characteristics, and Medicare HMO Performance. Health Care Financing Review 22(4):141-53.

Hoenig, H., L. Rubenstein, and K. Kahn. 1996. Rehabilitation after hip fracture: equal opportunity for all? Archives of Physical Medicine and Rehabilitation 77(1):58-63.

Hu, P., and D.B. Reuben. 2002. Effects of managed care on the length of time that elderly patients spend with physicians during ambulatory visits: National Ambulatory Medical Care Survey. Medical Care. 40(7):606-13.

Lau, R.R., and J.E. Ware. 1981. Refinements in the measurement in health-specific locus-ofcontrol beliefs. Medical Care 19:1147-1157.

Manning, W.G., and J. Mullahy. 2001. Estimating log models: to transform or not to transform? Journal of Health Economics 20:461-494.

Manning, W.G., C.N. Morris, and J.P. Newhouse, et al. 1981. A two-part model of the demand for medical care: preliminary results from the Health Insurance Study. In: van der Gaag J, Perlman M, eds. Health, Economics, and Health Economics. Amsterdam, Holland: North Holland: 103-123.

Manning, W.G., A. Leibowitz, and G.A. Goldberg, et al. 1984. A controlled trial of the effect of a prepaid group practice on use of services. New England Journal of Medicine 310:1505-1510.

Manning, W.G., J.P. Newhouse, N. Duan, E.B. Keeler, A. Leibowitz, and M.S. Marquis. 1987. Health insurance and the demand for medical care: evidence from a randomized experiment. American Economic Review 77:251-278.

Marshall, G.N., B.E. Collins, V.C. Crooks. 1990. A comparison of two multidimensional health locus of control instruments. Journal of Personality Assessment 54:181-190.

McCullagh, P., and J.A. Nelder. 1989. Generalized Linear Models, $2^{\text {nd }}$ Edition. London, England: Chapman \& Hall.

Miller, B., R.T. Campbell, and S. Furner, et al. 1997. Use of medical care by African American and white older persons: comparative analysis of three national data sets. Journal of Gerontology (Social Sciences) 52B(6): S325-S335. 
Miller, R.H., and H.S. Luft. 1997. Does managed care lead to better or worse quality of care? Health Affairs 16(5):7-25.

Miller, R.H., and H.S. Luft. 1994. Managed care plan performance since 1980: a literature analysis. Journal of the American Medical Association 271:1512-1519.

Mutchler, J.E., and J.A. Burr. 1991. Racial differences in health and health care service utilization in late life. Journal of Health and Social Behavior 32(4):342-356.

Physician Payment Review Commission. 1996. Access to Care in Medicare Managed Care: Results from a 1996 Survey of Enrollees and Disenrollees. Washington, D.C.: PPRC.

Sarver, J.H., R.K. Cydulka, and D.W. Baker. 2002. Usual Source of Care and Nonurgent Emergency Department Use. Academic Emergency Medicine 9 (9): 916-23.

Schneider, E.C., P.D. Cleary, A.M. Zaslavsky, and A.M. Epstein. 2001. Racial disparity in influenza vaccination: does managed care narrow the gap between African Americans and whites? Journal of the American Medical Association 286(12), 1455-60.

Schneider, E.C., A.M. Zaslavsky, A.M. Epstein. 2002. Racial disparities in the quality of care for enrollees in Medicare managed care. Journal of the American Medical Association 287(10):1288-94.

Scott, T.L., J.A. Gazmararian, M.V. Williams, and D.W. Baker. 2002. Health literacy and preventive health care use among Medicare enrollees in a managed care organization. Medical Care. 40(5): 395-404.

Smith, J. 1997. Wealth inequality among older Americans. Journal of Gerontology (Special Issue: Asset and Health Dynamics Among the Oldest Old [AHEAD]: Initial Results from the Longitudinal Study) 52B(Special Issue):74-81.

Stern, R.S., J.S. Weissman, and A.M. Epstein. 1991. The emergency department as a pathway to admission for poor and high cost patients. Journal of the American Medical Association. 266(16):2238-2243.

Tudor, C.G., G. Riley, and M. Ingber. 1998. Satisfaction with care: do Medicare HMOs make a difference? Health Affairs. 17(2):165-76.

Ware, J., M. Kosinski, and S.D. Keller. 1996. A 12-item short-form health survey: construction of scales and preliminary tests of reliability and validity. Medical Care 34(3), 220-33.

Williams, R.L., S.A. Flocke, and K.C. Stange. 2001. Race and preventive services delivery among black patients and white patients seen in primary care. Medical Care 39(11):1260-1267. 


\section{TABLE 1: SAMPLE CHARACTERISTICS}

\begin{tabular}{|c|c|}
\hline Variable & Mean \\
\hline \multicolumn{2}{|l|}{ Education } \\
\hline Less than high school & $38 \%$ \\
\hline High school graduate & $37 \%$ \\
\hline Some college & $25 \%$ \\
\hline \multicolumn{2}{|l|}{ Household Income } \\
\hline Poor $(<100 \%$ poverty $)$ & $11 \%$ \\
\hline Low income (100-200\% poverty) & $44 \%$ \\
\hline Middle income (200- $400 \%$ poverty) & $32 \%$ \\
\hline High income (400\% and up) & $13 \%$ \\
\hline \multicolumn{2}{|l|}{ Household Wealth } \\
\hline Low assets (1st tertile) & $33 \%$ \\
\hline Medium assets (2nd tertile) & $33 \%$ \\
\hline High assets (3rd tertile) & $33 \%$ \\
\hline \multicolumn{2}{|l|}{ Age } \\
\hline 65 to 69 & $25 \%$ \\
\hline 70 to 74 & $29 \%$ \\
\hline 75 to 79 & $23 \%$ \\
\hline 80 to 84 & $14 \%$ \\
\hline 85 or older & $8 \%$ \\
\hline \multicolumn{2}{|l|}{ Sex } \\
\hline Female & $56 \%$ \\
\hline Male & $44 \%$ \\
\hline \multicolumn{2}{|l|}{ Race/Ethnicity } \\
\hline White & $88 \%$ \\
\hline Black & $7 \%$ \\
\hline Other & $5 \%$ \\
\hline \multicolumn{2}{|l|}{ Marital Status } \\
\hline Married & $42 \%$ \\
\hline Widowed & $43 \%$ \\
\hline Divorced/Separated & $11 \%$ \\
\hline Never married & $4 \%$ \\
\hline \multicolumn{2}{|l|}{ Health Status } \\
\hline PCS score & 44.0 \\
\hline MCS score & 54.4 \\
\hline Ever smoked & $57 \%$ \\
\hline Self-rated health is Excellent/Very Good & $28 \%$ \\
\hline Self-rated health is Good & $41 \%$ \\
\hline Self-rated health is Fair & $27 \%$ \\
\hline Self-rated health is Poor & $4 \%$ \\
\hline High blood pressure & $57 \%$ \\
\hline Diabetes & $17 \%$ \\
\hline
\end{tabular}


TABLE 1: SAMPLE CHARACTERISTICS

\begin{tabular}{lr}
\hline Variable & Mean \\
\hline & \\
Cancer & $14 \%$ \\
Lung disease & $14 \%$ \\
Heart attack & $13 \%$ \\
CHF & $6 \%$ \\
Angina & $12 \%$ \\
Stroke & $9 \%$ \\
Arthritis & $54 \%$ \\
Ulcers & $12 \%$ \\
Bowel disease & $7 \%$ \\
Glaucoma & $9 \%$ \\
Depression & $11 \%$ \\
Weight loss & $4 \%$ \\
Died during year & $3 \%$ \\
& \\
Other & \\
Proxy status & $12 \%$ \\
Northeast plan & $69 \%$ \\
Dual Medicaid eligibility & $4 \%$ \\
& \\
\hline Number of Observations & 4600 \\
\hline
\end{tabular}

Note: All means are weighted. 
TABLE 2: MEANS OF PATHWAY VARIABLES

\begin{tabular}{lr}
\hline Variable & Mean \\
\hline & \\
Preferences & 6.0 \\
Care Attitudes (summary score, range 3-9) & $84 \%$ \\
Insured (18-64): All or most of the time & $16 \%$ \\
Insured (18-64):Some or none of the time & $75 \%$ \\
Never in HMO & $12 \%$ \\
In HMO less than 10 yrs & $13 \%$ \\
In HMO 10 or more years & $77 \%$ \\
Usual source of care (18-64): doctor/clinic & $8 \%$ \\
Usual source of care (18-64): other & $15 \%$ \\
Usual source of care (18-64): none & \\
& \\
Ability to Navigate the System & $26 \%$ \\
Belonged to HMO before study plan & 3.2 \\
Ease of appointment (1: very hard, 4 : very easy) & 3.2 \\
Ease of contact (1: very hard, $4:$ very easy) & $95 \%$ \\
Always got referral when needed & 2.5 \\
Self-rated memory (1:excellent, 5: poor) & 2.5 \\
Self-rated decisions (1:excellent, 5: poor) & $57 \%$ \\
Living alone & 2.9 \\
Number of children &
\end{tabular}


TABLE 3: PREDICTED EXPENDITURES, BY EXPENDITURE CATEGORY AND SOCIOECONOMIC STATUS

\section{Expenditure Category}

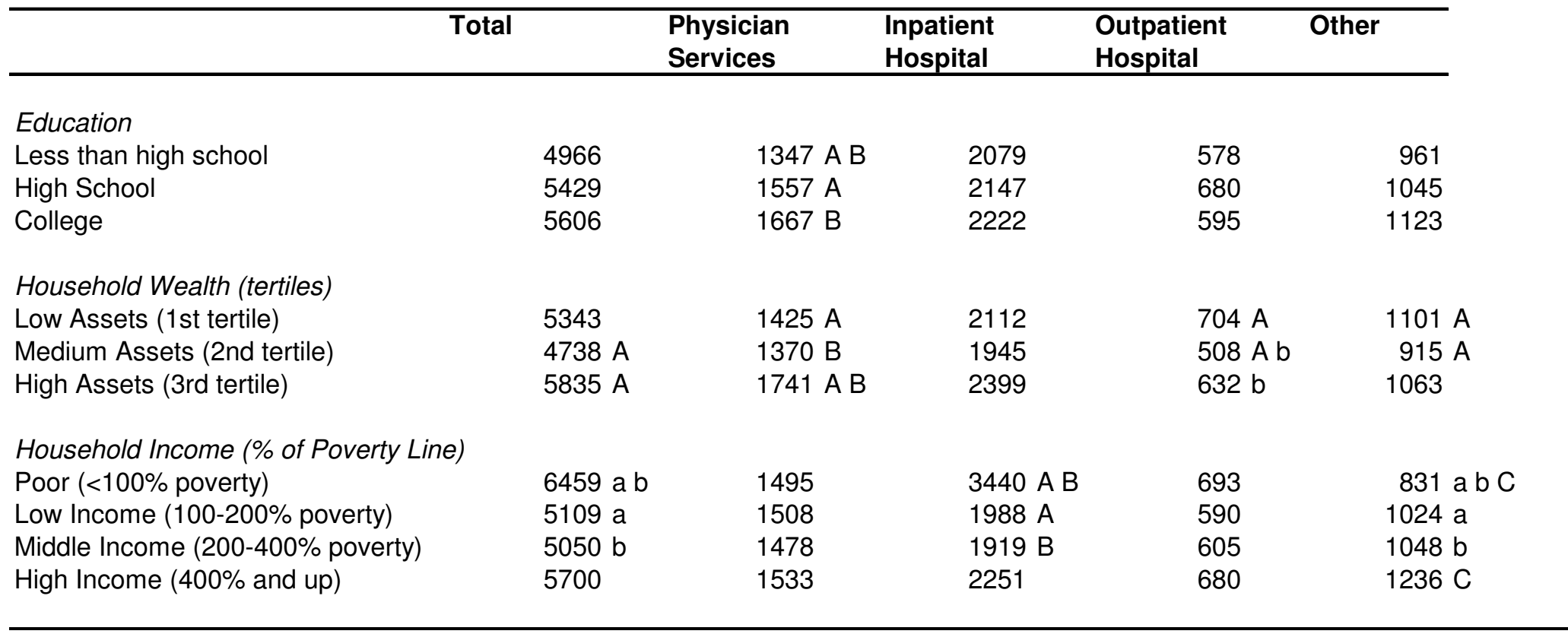

Note: Letters show statistical significance between pairs of predictions within an SES category.

Upper case letters indicate significance at the 5\% level; lower case letters indicate significance at the $10 \%$ level

For example: The poor have significantly higher total expenditure than the low income and the middle income (at the $10 \%$ level)

Multivariate analyses adjust for age, sex, race, marital status, health status (measured by PCS, MCS, self reported health and

chronic conditions), Medicaid enrollment, plan membership, proxy interview status, and death during the year. 
TABLE 4: PREDICTED PHYSICIAN EXPENDITURES, BY TYPE OF PHYSICIAN AND SOCIOECONOMIC STATUS

\begin{tabular}{llll}
\hline Total & Primary Care & Specialists \\
Physician & Physicians & \\
\hline
\end{tabular}

\section{Education}

Less than high school

High School

College

Household Wealth (tertiles)

Low Assets (1st tertile)

Medium Assets (2nd tertile)

High Assets (3rd tertile)

Household Income (\% of Poverty Line)

Poor $(<100 \%$ poverty)

Low Income (100-200\% poverty)

Middle Income (200-400\% poverty)

High Income (400\% and up)

\begin{tabular}{llc}
$1347 \mathrm{~A} \mathrm{~B}$ & 259 & $783 \mathrm{~A} \mathrm{~B}$ \\
$1557 \mathrm{~A}$ & 276 & $944 \mathrm{~B}$ \\
$1667 \mathrm{~B}$ & 283 & $977 \mathrm{~A}$ \\
& & \\
& & \\
$1425 \mathrm{~A}$ & $289 \mathrm{~A}$ & $805 \mathrm{~A}$ \\
$1370 \mathrm{~B}$ & $254 \mathrm{~A}$ & $831 \mathrm{~B}$ \\
$1741 \mathrm{~A} \mathrm{~B}$ & 267 & $1056 \mathrm{~A} \mathrm{~B}$ \\
& & \\
1495 & 277 & 842 \\
1508 & 264 & 905 \\
1478 & 275 & 872 \\
1533 & 281 & 916 \\
\hline
\end{tabular}

Note: Letters show statistical significance between pairs of predictions within an SES category.

Upper case letters indicate significance at the 5\% level; lower case letters indicate significance at the $10 \%$ level

Multivariate analyses adjust for age, sex, race, marital status, health status (measured by PCS, MCS, self reported health and chronic conditions), Medicaid enrollment, plan membership, proxy interview status, dual Medicaid eligibility, and death during the year. 
TABLE 5: MARGINAL EFFECTS OF PATHWAY VARIABLES ON MEDICAL CARE EXPENDITURES

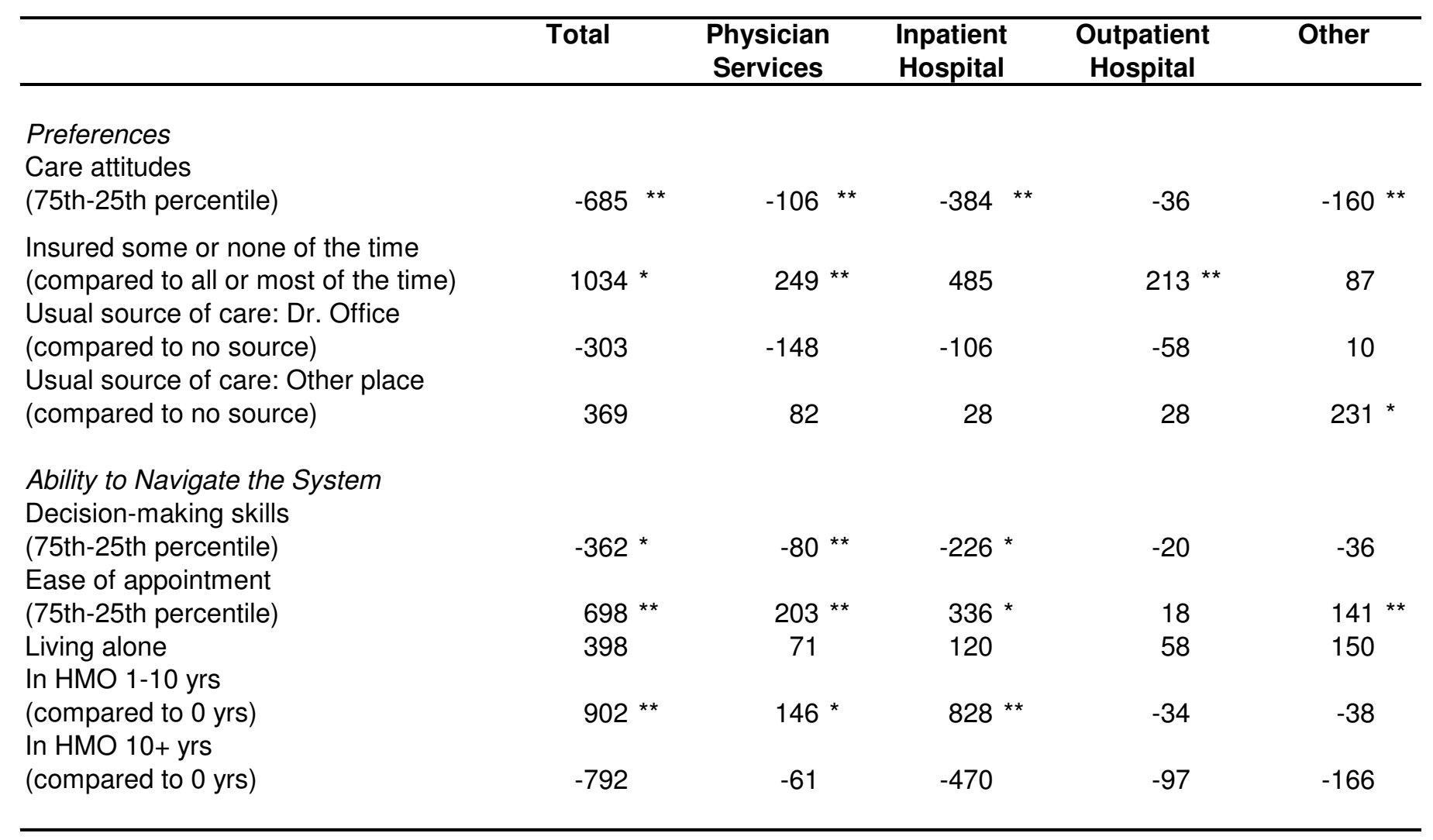

Note: * Denotes significance at $10 \%$ level ${ }^{* *}$ Denotes significance at $5 \%$ level 
TABLE 6: RATIO OF EXPENDITURES, BY SES CATEGORY AND EXPENDITURE TYPE, WITH AND WITHOUT ADJUSTING FOR PATHWAY VARIABLES

\begin{tabular}{|c|c|c|c|c|c|c|c|c|c|c|}
\hline \multirow[b]{2}{*}{ Pathway Variables } & \multicolumn{2}{|c|}{ Total } & \multicolumn{2}{|c|}{$\begin{array}{l}\text { Physician } \\
\text { Services }\end{array}$} & \multicolumn{2}{|c|}{$\begin{array}{l}\text { Inpatient } \\
\text { Hospital }\end{array}$} & \multicolumn{2}{|c|}{$\begin{array}{c}\text { Outpatient } \\
\text { Hospital }\end{array}$} & \multicolumn{2}{|c|}{ Other } \\
\hline & No & Yes & No & Yes & No & Yes & No & Yes & No & Yes \\
\hline \multicolumn{11}{|l|}{ Education } \\
\hline Less than high school & 1.00 & 1.00 & 1.00 & 1.00 & 1.00 & 1.00 & 1.00 & 1.00 & 1.00 & 1.00 \\
\hline High School & 1.09 & 1.06 & 1.16 & 1.13 & 1.03 & 1.00 & 1.18 & 1.17 & 1.09 & 1.04 \\
\hline College & 1.13 & 1.09 & 1.24 & 1.21 & 1.07 & 1.01 & 1.03 & 1.01 & 1.17 & 1.13 \\
\hline \multicolumn{11}{|l|}{ Household Income } \\
\hline Poor (<100\% poverty) & 1.00 & 1.00 & 1.00 & 1.00 & 1.00 & 1.00 & 1.00 & 1.00 & 1.00 & 1.00 \\
\hline Low Income (100-200\% poverty) & 0.79 & 0.81 & 1.01 & 1.01 & 0.58 & 0.61 & 0.85 & 0.85 & 1.23 & 1.21 \\
\hline Middle Income (200-400\% poverty) & 0.78 & 0.82 & 0.99 & 1.01 & 0.56 & 0.60 & 0.87 & 0.88 & 1.26 & 1.27 \\
\hline High Income (400\% and up) & 0.88 & 0.92 & 1.03 & 1.03 & 0.65 & 0.72 & 0.98 & 0.98 & 1.49 & 1.44 \\
\hline \multicolumn{11}{|l|}{ Household Wealth (tertiles) } \\
\hline Low Assets (1st tertile) & 1.00 & 1.00 & 1.00 & 1.00 & 1.00 & 1.00 & 1.00 & 1.00 & 1.00 & 1.00 \\
\hline Medium Assets (2nd tertile) & 0.89 & 0.88 & 0.96 & 0.97 & 0.92 & 0.90 & 0.72 & 0.72 & 0.83 & 0.83 \\
\hline High Assets (3rd tertile) & 1.09 & 1.10 & 1.22 & 1.21 & 1.14 & 1.17 & 0.90 & 0.89 & 0.97 & 0.97 \\
\hline
\end{tabular}

Note: Each value in the table is the ratio of predicted expenditures for enrollees in the particular education, income, or wealth category relative to enrollees in the reference category for that SES variable. For instance, the reference category for education is less than a high school degree. The columns labeled "No" are for models that did not include the pathway variables as covariates. Those labeled "Yes" are for models that adjusted for the pathway variables. Multivariate analyses adjust for age, sex, race, marital status, health status (measured by PCS, MCS, self reported health and chronic conditions), Medicaid enrollment, plan membership, proxy interview status, dual Medicaid eligibility, and death during the year. 


\begin{tabular}{|c|c|c|c|c|c|}
\hline & Total & Physician & Inpatient & Outpatient & Other \\
\hline \multicolumn{6}{|l|}{ Marital Status } \\
\hline Widowed & 375 & 158 & 54 & 104 & 59 \\
\hline Divorced/Separated & 71 & 214 & -311 & 80 & 87 \\
\hline Never Married & 978 & 147 & 607 & 85 & 139 \\
\hline \multicolumn{6}{|l|}{ Race } \\
\hline Black & 71 & -39 & 35 & 66 & 10 \\
\hline Other & -116 & 73 & -202 & 27 & -14 \\
\hline \multicolumn{6}{|l|}{ Health } \\
\hline $\begin{array}{l}\text { percentile) } \\
\text { MCS Score (75th -25th }\end{array}$ & $-2233 * *$ & -376 ** & $-1030 * *$ & $-288^{* *}$ & -538 ** \\
\hline percentile) & -147 & -63 & 27 & -47 & -65 \\
\hline Ever Smoked & 902 ** & $231^{* *}$ & 408 & 11 & $252^{* *}$ \\
\hline \multicolumn{6}{|c|}{$\begin{array}{l}\text { Self-reported health is good } \\
\text { (omitted category is }\end{array}$} \\
\hline excellent/very good) & 195 & 148 * & -83 & 17 & 112 \\
\hline Health is Fair & 544 & $421 * *$ & -164 & 67 & 220 * \\
\hline Health is Poor & 1764 & 582 ** & 609 & 281 & 291 \\
\hline High Blood Pressure & 513 & 56 & 367 & $104^{* *}$ & -14 \\
\hline Diabetes & 676 & -6 & 312 & 54 & 318 ** \\
\hline Cancer & 1802 ** & $706^{* *}$ & 173 & 152 * & $771^{* *}$ \\
\hline Lung Disease & 946 * & -96 & $937^{* *}$ & -19 & 124 \\
\hline Heart Attack & -298 & 26 & -71 & -5 & -248 ** \\
\hline $\mathrm{CHF}$ & 1505 * & $509 * *$ & 611 & 193 & 192 \\
\hline Angina & 1028 * & 460 ** & 413 & 19 & 136 \\
\hline Stroke & 807 & 55 & 457 & 169 * & 127 \\
\hline Arthritis & 256 & 216 ** & 44 & -28 & 23 \\
\hline Ulcers & 995 & $240 *$ & 558 & 41 & 156 \\
\hline Bowel Disease & -176 & 9 & -299 & 95 & 19 \\
\hline Glaucoma & 168 & 172 & -120 & 109 & 7 \\
\hline Depression & 1003 * & 327 * & 467 & 132 & 77 \\
\hline Weight Loss & 3327 ** & 406 * & 1837 * & 316 * & 768 * \\
\hline \multicolumn{6}{|c|}{ Demographics } \\
\hline \multicolumn{6}{|c|}{ Age: 70 to 74 (omitted category } \\
\hline is 65 to 69$)$ & 413 & 272 ** & -64 & 94 & 112 \\
\hline Age: 75 to 79 & 294 & 188 * & 66 & 51 & -10 \\
\hline Age: 80 to 84 & 2285 ** & $466^{* *}$ & 1099 ** & 519 ** & 201 \\
\hline Age: 85 plus & 1115 * & 207 & 340 & 148 & 419 ** \\
\hline Female & -1569 ** & -320 ** & $-1027^{* *}$ & $-181^{* *}$ & -40 \\
\hline Died during year & 20796 ** & 3704 ** & 13358 ** & $1037^{* *}$ & $2698^{* *}$ \\
\hline \multicolumn{6}{|l|}{ Plan Variables } \\
\hline Proxy status & -1129 ** & $-301 * *$ & -532 * & $-149 * *$ & -147 \\
\hline Northeast Plan & -20 & -37 & -305 & $117^{* *}$ & 205 ** \\
\hline Medicaid & 284 & -36 & 243 & 13 & 64 \\
\hline
\end{tabular}

${ }^{* *}$ indicates significance at the $5 \%$ level; * indicates significance at the $10 \%$ level 